La revue La revue pour l'histoire du CNRS

POUR L'HISTOIRE DU CNRS $\quad 2$ | 2000

Les premiers laboratoires du CNRS

\title{
La fondation Rockefeller et la recherche médicale
}

Jean-François Picard, Presses universitaires de France, Paris, 1999

Muriel Le Roux

\section{CpenEdition}

Journals

Édition électronique

URL : https://journals.openedition.org/histoire-cnrs/379

DOI : 10.4000/histoire-cnrs.379

ISSN : 1955-2408

Éditeur

CNRS Éditions

Édition imprimée

Date de publication : 5 mai 2000

ISBN : 978-2-271-05708-2

ISSN : $1298-9800$

Référence électronique

Muriel Le Roux, "La fondation Rockefeller et la recherche médicale », La revue pour l'histoire du CNRS

[En ligne], 2 | 2000, mis en ligne le 06 mars 2006, consulté le 20 mai 2021. URL : http://

journals.openedition.org/histoire-cnrs/379; DOI : https://doi.org/10.4000/histoire-cnrs.379

Ce document a été généré automatiquement le 20 mai 2021

Comité pour l'histoire du CNRS 


\title{
La fondation Rockefeller et la recherche médicale
}

\author{
Jean-François Picard, Presses universitaires de France, Paris, 1999
}

\section{Muriel Le Roux}

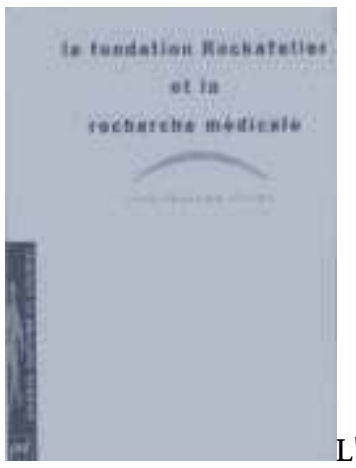

L'histoire de la médecine s'est enrichie d'un nouvel ouvrage complétant ceux, pour ne citer que quelques auteurs, de Jean-Paul Gaudillière, Michel Morange, Claire Salomon-Bayet et pour le secteur pharmaceutique ceux de Michèle Ruffat et de Sophie Chauveau. Celui de Jean-François Picard se focalise sur la recherche médicale dont la genèse nous est présentée grâce à la reconstitution de l'action menée par la fondation américaine Rockefeller. L'auteur se place au niveau des décideurs de la philanthropie américaine, rappelant les méthodes de l'histoire des entreprises. Ce qui n'est pas neutre lorsqu'il s'agit de la fondation Rockefeller. La période considérée présente le système sanitaire, social et médical américain de la fin du XIX ${ }^{\mathrm{e}}$ siècle et nous conduit aux années 50 de notre siècle, époque d'«homogénéisation», de standardisation des organisations de la recherche scientifique, d'américanisation, écrit l'auteur. C'est à cette époque que l'on commence aussi à contester la distance trop grande qui existe entre la médecine qui cherche et celle qui soigne, processus qui conduit à déshumaniser la médecine.

1 La philanthropie de Rockefeller s'appuie de façon permanente sur des états des lieux, des enquêtes (état sanitaire des populations), des évaluations de la qualité des enseignements, des formations puis des programmes. Ce fut à la suite d'enquêtes (1910 et 1912) ordonnées par l'autre grande fondation, la « Carnegie », sur l'enseignement de 
la médecine aux États-Unis et en Europe (Allemagne, France, Grande-Bretagne) que le "mécène utilitariste", John Davison Rockefeller, magnat du pétrole, mena des réflexions qui furent à l'origine de la création de l'institut de recherche médical du même nom. L'opposition de l'opinion publique américaine aux pratiques des barons voleurs fut si vive que l'empire pétrolier de Rockefeller fut démantelé en 1911. Aussi, pour s'amender, J. D. Rockefeller créa, en 1913, la fondation dont il est ici question et y intégra l'institut.

2 En France, tout comme en Grande-Bretagne, l'hôpital était le lieu privilégié de l'enseignement clinique. L'Allemagne, comme dans d'autres secteurs scientifiques sut associer la méthode analytique, privilégier l'enseignement dans les facultés des sciences, soutint la recherche en laboratoire. C'est dans ce pays, le premier à avoir considéré la recherche comme une affaire d'État, qu'est née la médecine moderne alliant observation clinique et recherche scientifique. Les Rockefeller surent toujours s'entourer de conseillers avisés, utilisèrent le principe des enquêteurs pour s'informer en permanence de ce qui prévalait sur le vieux continent, mais aussi dans le reste du monde à mesure que le temps passait.

Et l'on retrouve, ici, la façon dont les Américains, qu'il s'agisse de science, de technique ou d'industrie, sont venus étudier avec profit en Europe au cours du XIX siècle pour créer chez eux universités, instituts, centres de recherche médicaux et industriels. Ainsi l'Institut de recherche médical Rockefeller s'inspire directement de l'Institut Pasteur et de l'Institut Koch à Berlin. Certaines universités américaines bénéficièrent ou furent créées grâce aux philanthropes en s'inspirant du modèle européen.

4 La Première Guerre mondiale renversa la tendance au profit de la recherche médicale américaine. L'institut consacra ses premières recherches à la chimie et à la biologie affirmant clairement que la recherche fondamentale devait servir au progrès de la médecine. La jeune Fondation se consacrait à la réforme de l'enseignement de la médecine qui reposait sur le principe d'une intégration des hôpitaux au système des facultés de médecine, avec des enseignants à plein temps. Ce full time des praticiens fut l'enjeu de la réforme aux États-Unis, mais aussi en France après la Seconde Guerre mondiale. Le conservatisme du corps médical américain et français, réticent aussi bien à l'introduction de la recherche qu'aux réformes universitaires, rappelle que l'introduction de toute innovation (scientifique ou organisationnelle) crée des conflits tant les tensions qui l'accompagnent sont fortes. Les réformes de Robert Debré établirent un compromis qui maintint des lits privés au sein des hôpitaux.

5 Ces principes acquis aux États-Unis, ce fut à l'étranger que la philanthropie de la Fondation s'exerça tout en restant fidèle à la double orientation. Elle distribuait les aides en fonction des besoins après enquêtes menées par les Rockefeller officers. L'aide prit différentes formes dont la distribution de bourses aux meilleurs étudiants en médecine européens ou aux jeunes chercheurs prometteurs qui travaillaient sur des sujets nouveaux et dans des disciplines nouvelles. L'aide a aussi été fournie sous forme d'allocations à des organismes privilégiant les recherches fondamentales dans des disciplines nouvelles (biologie moléculaire). Les États concernés devaient investir à concurrence de la dotation Rockefeller. Cette obligation mutuelle explique que la fondation ait continué à envoyer des subsides en Allemagne jusqu'à la veille de l'entrée en guerre des États-Unis. Il y eut aussi des dotations sous formes d'équipement et d'instruments... L'évaluation de la qualité scientifique des projets et la valeur des 
individus furent toujours les principaux critères de sélection au-delà des opinions politiques. Ce qui a été reproché à la Fondation.

6 Le progrès de la science et sa diffusion auprès du plus grand nombre ont été les principales motivations des Rockefeller. Il y eut de grands succès, des échecs, notamment celui de la modernisation de la faculté de médecine de Paris. Tous les centres de recherche européens ont peu ou prou bénéficié de la manne financière. Ainsi la Rockefeller a aussi bien agi sur l'organisation des sciences, les principes d'évaluation des programmes de recherche et d'enseignement. Elle se heurtait souvent aux différentes manifestations des intérêts et des fiertés nationales. Au total, son influence, quelque fois contestée, n'en est pas moins réelle et incontestable.

7 Il reste à mener au cas par cas des études sur les différents réseaux créés ou «alimentés " par la fondation, réseaux scientifiques -par discipline en recherche et dans l'enseignement-, réseaux humains, réseaux technologiques -dans quelle mesure la fondation a contribué à la conception de nouveaux équipements-, réseaux politiques nationaux, internationaux. Des questions se posent. Dans quelle mesure, par exemple, les structures de la Fondation, l'organisation des divisions, les modalités de l'information accumulées (les enquêtes, les observations) par les Rockefeller officers, les bourses accordées aux Européens auraient-elles servi de modèles aux promoteurs de l'aide Marshall ? Il faudra aussi songer à écrire l'histoire de la Fondation mais du côté des médecins et des chercheurs anonymes, voire des malades pour savoir si la présence de la science depuis la prise en charge des patients jusqu'à la fin de la thérapie, à contribué à « déshumaniser » la médecine...

Quel a été l'impact de la Fondation dans la politique des sciences des différents États et organes bénéficiaires - dont le CNRS. Là encore, il faut poursuivre cette recherche, car seule une étude chiffrée et comparée permettra de donner la réponse. On le voit, il reste de vastes chantiers à investir pour écrire l'histoire des sciences... Une chose est sûre, le genre monographie est un moyen d'y parvenir.

\section{AUTEUR}

\section{MURIEL LE ROUX}

Chargée de recherche IHMC - CNRS 